Tropical Journal of Pharmaceutical Research, March 2016; 15 (3): 591-598

ISSN: $1596-5996$ (print); 1596-9827 (electronic)

(C) Pharmacotherapy Group, Faculty of Pharmacy, University of Benin, Benin City, 300001 Nigeria.

All rights reserved.

Available online at http://www.tjpr.org

Original Research Article

http://dx.doi.org/10.4314/tjpr.v15i3.22

\title{
Synthesis, Characterization, Antibacterial, $\alpha$-Glucosidase Inhibition and Hemolytic Studies on Some New N-(2,3- Dimethylphenyl)benzenesulfonamide Derivatives
}

\author{
Muhammad A Abbasi ${ }^{1 \star}$, Mudassar Islam ${ }^{1}$, Aziz-ur-Rehman', Shahid Rasool ${ }^{1}$, \\ Kaniz Rubab1, Ghulam Hussain1, Irshad Ahmad', Muhammad Ashraf ${ }^{3}$, \\ Muhammad Shahid ${ }^{4}$ and Syed Adnan Ali Shah ${ }^{5,6}$
}

${ }^{1}$ Department of Chemistry, Government College University, Lahore-54000, ${ }^{2}$ Department of Pharmacy, ${ }^{3}$ Department of, Chemistry, The Islamia University of Bahawalpur ,Bahawalpur-63100, ${ }^{4}$ Department of Chemistry and Biochemistry, University of Agriculture, Faisalabad-38040, Pakistan, ${ }^{5}$ Faculty of Pharmacy, Universiti Teknologi MARA, Puncak Alam-42300, ${ }^{6}$ Atta-urRahman Institute for Natural Products Discovery (AuRIns), Level 9, FF3, Universiti Teknologi MARA, Puncak Alam Campus, Bandar Puncak Alam, Selangor Darul Ehsan, Malaysia

*For correspondence: Email: atrabbasi@yahoo.com, abbasi@gcu.edu.pk; Tel: (+92)-42-111000010 ext 266.

Revised accepted: 5 January 2016

\begin{abstract}
Purpose: To synthesize a series of new N-(2,3-dimethylphenyl)benzenesulfonamide derivatives with pharmacological analysis.

Methods: N-(2,3-Dimethylphenyl)benzenesulfonamide (3) was synthesized by the reaction between 2,3-dimethylaniline (1) and benzenesulfonyl chloride (2) in aqueous basic medium. Compound 3 was further treated with various alkyl/aralakyl halides (4a-m) to yield new compounds, 5a-m, in a weak basic aprotic polar organic medium. The proposed structures of synthesized compounds were confirmed using proton-nuclear magnetic resonance (1H-NMR), infra-red spectroscopy (IR) and electron impact mass spectrometry (EIMS). The synthesized compounds were screened for in vitro antibacterial, antienzymatic and hemolytic activities using standard procedures.

Results: All the synthesized compounds showed moderate to high activity against Gram-positive and Gram-negative bacterial strains. The molecules $5 g$ and $5 j$ exhibited good inhibition of $\alpha$-glucosidase enzyme with half-maximal inhibitory concentration $\left(I C_{50}\right)$ of $59.53 \pm 0.01$ and $55.31 \pm 0.01 \mu \mathrm{moles} / L$, respectively, relative to acarbose with $I C_{50}$ of $38.25 \pm 0.12 \mu \mathrm{moles} / \mathrm{L}$. All the compounds exhibited cytotoxicity levels ranging from $27.20 \pm 0.24$ to $5.20 \pm 0.41 \%$, relative to Triton $X-100$.

Conclusion: Compound $5 f$ is the most potent antibacterial while $5 j$ is the best $\alpha$-glucosidase inhibitor; 5e showed the least cytotoxicity.
\end{abstract}

Keywords: 2,3-Dimethylaniline, Antibacterial activity, Anti-enzymatic activity, a-Glucosidase inhibitor, Hemolytic activity, Sulfonamides

Tropical Journal of Pharmaceutical Research is indexed by Science Citation Index (SciSearch), Scopus, International Pharmaceutical Abstract, Chemical Abstracts, Embase, Index Copernicus, EBSCO, African Index Medicus, JournalSeek, Journal Citation Reports/Science Edition, Directory of Open Access Journals (DOAJ), African Journal Online, Bioline International, Open-J-Gate and Pharmacy Abstracts

\section{INTRODUCTION}

Sulfonamides exhibit numerous pharmacological activities [1-3] and sulfonamide antibiotics are among the most common instigators of allergic or hypersensitivity reactions [4-6]. $\alpha$-Glucosidase
( $\alpha$-D-glucosideglucohydrolase,

EC 3.2.1.20)hydrolyzes the 1,4-glycosidic linkage from the non-reducing end of the a-glucosides [7-9].Its inhibitors retard the liberation of Dglucose resulting in delay glucose absorption [10]. The hemolysis takes place due to the stroke 
of the microbial products and resident parasites on the red blood corpuscles membranes [11,12]. Our group has synthesized many antibacterial and anti-enzymatic potent molecules [13-16] and a series of new compounds was synthesized in the present study to explore their pharmacological behavior.

\section{EXPERIMENTAL}

\section{General}

The chemicals solvents were of commercial grade and were used without purification. Reaction progress and product purity was monitored by pre-coated TLC silica gel G-25UV254 plates with different solvent systems using ethyl acetate and n-hexane. Melting points were noted on Gallenkamp apparatus by open capillary tube and were uncorrected. FTIR spectra were recorded on MIDAC M 2000 spectrometer. 1H-NMR spectra were recorded in $\mathrm{CDCl}_{3}$ on Bruker $(400 \mathrm{MHz})$. Mass spectra (ElMS) were measured on Finnigan MAT-312 instruments.

Procedure for the synthesis of $\mathrm{N}-(2,3-$ dimethylphenyl)benzenesulfonamide (3)

An equimolar mixture of 2,3-dimethylaniline (1, $0.02 \mathrm{~mol}$ ) was dispersed in $25 \mathrm{~mL}$ waterfollowed by the addition ofbenzenesulfonyl chloride (2, $0.02 \mathrm{~mol}$ ). The $\mathrm{pH}$ of the suspension was maintained at 9-10 during the whole reaction by adding $\mathrm{Na}_{2} \mathrm{CO}_{3}$ at $\mathrm{RT}$. The reaction solution was stirred for $3 \mathrm{~h}$. After complete reaction, the concentrated $\mathrm{HCl}$ (2-3 $\mathrm{mL}$ ) was added slowly to adjust the $\mathrm{pH}$ to 2 . Product formation was confirmed by TLC. The reaction mixture was kept still for 3-5 min. The off-white precipitates were collected by filtration, washed with distilled water and dried to acquire the compound 3 .

General procedure for the synthesis of $\mathrm{N}$ alkyl/aralakyl substituted sulfonamides (5am)

The calculated amount of $3(0.7 \mathrm{mmol} ; 0.2 \mathrm{~g})$ was taken in a round bottom flask $(50 \mathrm{~mL})$, then $\mathrm{N}, \mathrm{N}$-dimethyl formamide (DMF) was added to dissolve it followed by the addition of lithium hydride $\mathrm{LiH}(0.004 \mathrm{~g})$. The mixture was stirred for 45 min at RT and then alkyl/aralakyl halides (4a$\mathbf{m} ; 0.7 \mathrm{mmol}$ ) were added to the mixture. The solution was further stirred for 3-4 h. The progress of reaction was monitored by TLC. The product was precipitated by adding cold water, filtered, washed with distilled water and dried.

\section{Evaluation of antibacterial activity}

The antibacterial activity was performed in sterile 96-wells microplates under aseptic environments by the methods of Kaspady et al [17] and Yang et al [18].

\section{$\alpha$-Glucosidase assay}

The $\alpha$-glucosidase inhibition activity was performed according to the method of Chapdilaine et al [10].

\section{Determination of hemolytic activity}

Hemolytic activity was studied by the reported method of Powell et al $[11,19]$. $3.0 \mathrm{~mL}$ fresh heparin added human blood obtained from volunteers after guidance from the Department of Clinical Medicine and Surgery, University of Agriculture, Faisalabad, Pakistan. The study protocol was approved by the institutional ethical committee (approval no. DGS/8786-89, dated 09-03-2015), University of Agriculture, Faisalabad, Pakistan and was conducted in accordance with 1964 Declaration of Helsinki and its subsequent amendments [20].

\section{Data analysis}

All the measurements were performed in triplicate and statistical analysis was carried out by Microsoft Excel 2010. The results are presented as mean \pm SEM with CL $80 \%$ (for antibacterial), $85 \%$ (for $\alpha$-glucosidase) and $95 \%$ (for hemolytic analysis). MIC (minimum inhibitory concentration and $I_{50} \quad(50 \%$ inhibitory concentration) were calculated using EZ-Fitz software (Perrella Scientific Inc. Amherst, USA).

\section{RESULTS}

The new $\mathrm{N}$-substituted derivatives of $\mathrm{N}$-(2,3dimethylphenyl)benzenesulfonamide were synthesized according to the protocol sketched in Scheme-1.

The antibacterial activities are given in Table-1 while inhibition against $\alpha$-glucosidase enzyme and hemolytic activity are tabulated in Table 2.

\section{Spectral characteristics of synthesized compounds $(3,5 a-m)$}

\section{$\mathrm{N}$-(2,3-Dimethylphenyl)benzenesulfonamide} (3)

Yield: $98 \%$; off-white solid; mp. 118-119 ${ }^{\circ} \mathrm{C}$; Molecular formula: $\mathrm{C}_{14} \mathrm{H}_{15} \mathrm{NO}_{2} \mathrm{~S}$; Molecular 
<smiles>Cc1cccc(N)c1C</smiles>

(1)<smiles>O=S(=O)(Cl)c1ccccc1</smiles>

(2)

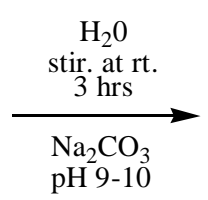

$\mathrm{pH}$ 9-10
(3)<smiles>Cc1cccc(NS(=O)(=O)c2ccccc2)c1C</smiles>

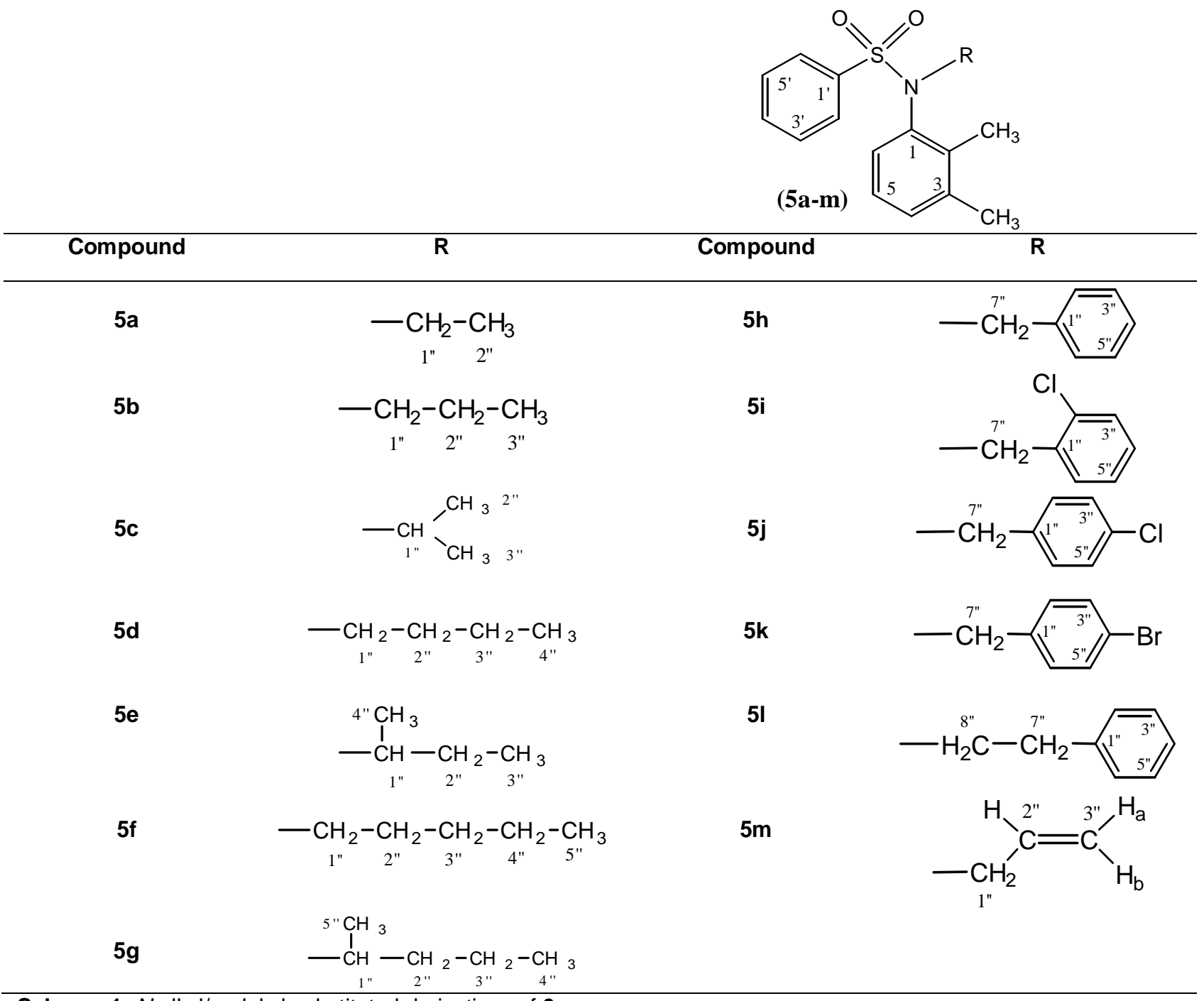

Scheme-1: $N$-alkyl/aralakyl substituted derivatives of $\mathbf{3}$

weight: 261; Analysis. Calcd: C, 64.32; H, 5.74; $\mathrm{N}, 5.36 ; \mathrm{S}, 12.25$. Found: C, 64.03; H, 6.40; N, 5.52; S, 11.73; IR $\left(\mathrm{cm}^{-1}\right) u_{\max }: 3208(\mathrm{~N}-\mathrm{H}), 3042(\mathrm{Ar}$ $\mathrm{C}-\mathrm{H}$ ), 2924(Alkane $\mathrm{C}-\mathrm{H}$ ), 1583( $\mathrm{Ar} \mathrm{C}=\mathrm{C}$ ), 1324 $(\mathrm{S}=\mathrm{O}) ;{ }^{1} \mathrm{H}-\mathrm{NMR}\left(400 \mathrm{MHz}, \mathrm{CDCl}_{3}, \delta\right.$ in $\left.\mathrm{ppm}\right)$ : $7.69\left(2 \mathrm{H}, d, J=7.6 \mathrm{~Hz}, \mathrm{H}-2^{\prime}, \mathrm{H}-6{ }^{\prime}\right), 7.53(1 \mathrm{H}, t, J$ $\left.=7.6 \mathrm{~Hz}, \mathrm{H}-4^{\prime}\right), 7.41\left(2 \mathrm{H}, t, J=7.6 \mathrm{~Hz}, \mathrm{H}-3^{\prime}, \mathrm{H}-\right.$ $\left.5^{\prime}\right), 7.13(1 \mathrm{H}, d, J=7.6 \mathrm{~Hz}, \mathrm{H}-6), 6.96(1 \mathrm{H}, t, J=$ 7.6Hz, H-5), $6.46(1 \mathrm{H}, d, J=8.4 \mathrm{~Hz}, \mathrm{H}-4), 2.18$ $\left(3 \mathrm{H}, s, \mathrm{CH}_{3}-2\right), 1.91\left(3 \mathrm{H}, s, \mathrm{CH}_{3}-3\right)$; El-MS $(\mathrm{m} / \mathrm{z})$ :
$261\left(\mathrm{M}^{+}, 28.2 \%\right), 141,120(\mathrm{BP}, 100 \%), 105,77$, 51.

\section{$\mathrm{N}$-(2,3-Dimethylphenyl)- $\mathrm{N}$-ethylbenzenesulfo- namide $(5 a)$}

Yield: $99 \%$; light mustard solid; mp. $85.8{ }^{\circ} \mathrm{C}$; Molecular formula: $\mathrm{C}_{16} \mathrm{H}_{19} \mathrm{NO}_{2} \mathrm{~S}$; Molecular weight: 289; Analysis. Calcd: C, 66.43; H, 6.56; $\mathrm{N}, 4.84 ; \mathrm{S}, 11.07$. Found: C, 64.90; H, 6.29; N, 
4.52; S, 11.02; IR (cm $\left.{ }^{-1}\right) U_{\max }: 3065$ (Ar C-H), 2934 (Alkane C-H), 1583 ( $\operatorname{Ar} \mathrm{C}=\mathrm{C}), 1341$ (S=O); ${ }^{1} \mathrm{H}-$ NMR (400 MHz, $\mathrm{CDCl}_{3}, \delta$ in ppm): $7.67(2 \mathrm{H}, d, J$ $=7.6 \mathrm{~Hz}, \mathrm{H}-2 ', \mathrm{H}-6 '), 7.55\left(1 \mathrm{H}, t, J=7.6 \mathrm{~Hz}, \mathrm{H}-4{ }^{\prime}\right)$, $7.46\left(2 \mathrm{H}, t, J=7.6 \mathrm{~Hz}, \mathrm{H}-3^{\prime}, \mathrm{H}-5 '\right), 7.08(1 \mathrm{H}, d, J$ $=7.6 \mathrm{~Hz}, \mathrm{H}-6), 6.92(1 \mathrm{H}, t, J=7.6 \mathrm{~Hz}, \mathrm{H}-5), 6.39$ $(1 \mathrm{H}, d, J=8.0 \mathrm{~Hz}, 1 \mathrm{H}, \mathrm{H}-4), 3.86-3.77(1 \mathrm{H}, m$, $\left.\mathrm{H}_{\mathrm{a}}-1 "\right)$, 3.31-3.23 (1H, m, $\left.\mathrm{H}_{\mathrm{b}}-1 "\right), 2.28(3 \mathrm{H}, s$, $\left.\mathrm{CH}_{3}-2\right), 2.26\left(3 \mathrm{H}, s, \mathrm{CH}_{3}-3\right), 1.02(3 \mathrm{H}, t, J=$ 7.2Hz, $\left.\mathrm{CH}_{3}-2^{\prime \prime}\right)$; El-MS (m/z): $289\left(\mathrm{M}^{+}, 9.3 \%\right)$, 148 (BP, 100\%), 141, 120, 105, 77, 51.

\section{$N$-(2,3-Dimethylphenyl)- $N$-propan-1- ylbenzenesulfonamide (5b)}

Yield: $99 \%$; light pink solid; $\mathrm{mp} .79{ }^{\circ} \mathrm{C}$; Molecular formula: $\mathrm{C}_{17} \mathrm{H}_{21} \mathrm{NO}_{2} \mathrm{~S}$; Molecular weight: 303; Analysis. Calcd: C, 67.32; H, 6.93; N, 4.62; S, 10.56. Found: $\mathrm{C}, 66.35 ; \mathrm{H}, 6.70 ; \mathrm{N}, 4.61$; $\mathrm{S}$, 10.57; IR $\left(\mathrm{cm}^{-1}\right) \quad U_{\max }: 3071 \quad(\mathrm{Ar} \mathrm{C}-\mathrm{H}), 2978$ (Alkane C-H), 1586 ( $\mathrm{Ar} \mathrm{C}=\mathrm{C}), 1336(\mathrm{~S}=\mathrm{O}) ;{ }^{1} \mathrm{H}-$ NMR (400 MHz, $\mathrm{CDCl}_{3}, \delta$ in ppm): $7.66(2 \mathrm{H}, d, J$ $\left.=7.2 \mathrm{~Hz}, \mathrm{H}-2 ', \mathrm{H}-66^{\prime}\right), 7.56\left(1 \mathrm{H}, t, J=7.6 \mathrm{~Hz}, \mathrm{H}-4{ }^{\prime}\right)$, $7.46\left(2 \mathrm{H}, t, J=7.6 \mathrm{~Hz}, 2 \mathrm{H}, \mathrm{H}-3^{\prime}, \mathrm{H}-5{ }^{\prime}\right), 7.08(1 \mathrm{H}$, $d, J=7.6 \mathrm{~Hz}, \mathrm{H}-6), 6.91(1 \mathrm{H}, t, J=7.6 \mathrm{~Hz}, \mathrm{H}-5)$, $6.89(1 \mathrm{H}, d, J=8.0 \mathrm{~Hz}, \mathrm{H}-4), 3.69-3.62(1 \mathrm{H}, m$, $\left.\mathrm{H}_{\mathrm{a}}-1 "\right), 3.18-3.12\left(1 \mathrm{H}, m, \mathrm{H}_{\mathrm{b}}-1 "\right), 2.27(3 \mathrm{H}, s$, $\left.\mathrm{CH}_{3}-2\right), 2.25\left(3 \mathrm{H}, s, \mathrm{CH}_{3}-3\right), 1.53-1.33(2 \mathrm{H}, m, \mathrm{H}-$ 2"), $0.83\left(3 \mathrm{H}, t, J=7.2 \mathrm{~Hz}, \mathrm{CH}_{3}-3 "\right)$; El-MS (m/z): $303\left(\mathrm{M}^{+}, 12.0 \%\right), 141,120$ (BP, 100\%), 105, 77, 51.

\section{$\mathrm{N}$-(2,3-Dimethylphenyl)-N-(1-methylethyl) benzenesulfonamide (5c)}

Yield: $53 \%$; light pink solid; mp. $103.1{ }^{\circ} \mathrm{C}$; Molecular formula: $\mathrm{C}_{17} \mathrm{H}_{21} \mathrm{NO}_{2} \mathrm{~S}$; Molecular weight: 303; Analysis. Calcd: C, 67.32; H, 6.93; N, 4.62; S, 10.56. Found: C, 66.56; H, 6.69; N, 4.37; S, 10.70; IR $\left(\mathrm{cm}^{-1}\right) U_{\max }: 3066(\mathrm{Ar} \mathrm{C}-\mathrm{H})$, 2977 (Alkane C-H), 1584 ( $\operatorname{Ar} \mathrm{C}=\mathrm{C}), 1335$ (S=O);

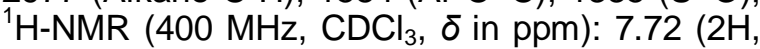
$\left.d, J=7.6 \mathrm{~Hz}, \mathrm{H}-2^{\prime}, \mathrm{H}-6^{\prime}\right), 7.53(1 \mathrm{H}, t, J=7.6 \mathrm{~Hz}$, $\left.\mathrm{H}-4^{\prime}\right), 7.44\left(2 \mathrm{H}, t, J=7.2 \mathrm{~Hz}, \mathrm{H}-3^{\prime}, \mathrm{H}-5^{\prime}\right), 7.13$ $(1 \mathrm{H}, d, J=7.6 \mathrm{~Hz}, \mathrm{H}-6), 6.96(1 \mathrm{H}, t, J=7.6 \mathrm{~Hz}, \mathrm{H}-$ 5), $6.56(1 \mathrm{H}, d, J=7.9 \mathrm{~Hz}, \mathrm{H}-4), 4.63-4.56(1 \mathrm{H}$, $m, \mathrm{H}-1 "), 2.28\left(3 \mathrm{H}, s, \mathrm{CH}_{3}-2\right), 2.23\left(3 \mathrm{H}, s, \mathrm{CH}_{3}-\right.$ 3), $1.02\left(3 \mathrm{H}, d, J=6.4 \mathrm{~Hz}, \mathrm{CH}_{3}-2 "\right), 0.92(3 \mathrm{H}, d, J$ $\left.=6.4 \mathrm{~Hz}, \mathrm{CH}_{3}-3 "\right)$; El-MS $(\mathrm{m} / \mathrm{z}): 303\left(\mathrm{M}^{+}, 33.4 \%\right)$, 141, 120 (BP, 100\%), 105, 77, 51.

\section{N-(2,3-Dimethylphenyl)-N-butan-1-ylbenzene- sulfonamide (5d)}

Yield: $98 \%$; light pink solid; mp. $54.7{ }^{\circ} \mathrm{C}$; Molecular formula: $\mathrm{C}_{18} \mathrm{H}_{23} \mathrm{NO}_{2} \mathrm{~S}$; Molecular weight: 317; Analysis. Calcd: C, 68.13; H, 7.25; $\mathrm{N}, 4.41 ; \mathrm{S}, 10.09$. Found: C, 66.91; H, 6.87; N, 4.09; S, 10.18; IR $\left(\mathrm{cm}^{-1}\right) \quad U_{\max }: 3070$ (Ar C-H), 2955 (Alkane C-H), 1583 ( $\mathrm{Ar} \mathrm{C}=\mathrm{C}), 1335$ (S=O);
${ }^{1} \mathrm{H}-\mathrm{NMR}\left(400 \mathrm{MHz}, \mathrm{CDCl}_{3}, \delta\right.$ in ppm): $7.65(2 \mathrm{H}$, $d, J=7.2 \mathrm{~Hz}, 2 \mathrm{H}, \mathrm{H}-2 ', \mathrm{H}-6 '), 7.56(1 \mathrm{H}, t, J=$ $\left.7.6 \mathrm{~Hz}, \mathrm{H}-4^{\prime}\right), 7.45\left(2 \mathrm{H}, t, J=7.6 \mathrm{~Hz}, \mathrm{H}-3^{\prime}, \mathrm{H}-5^{\prime}\right)$, $7.08(1 \mathrm{H}, d, J=7.2 \mathrm{~Hz}, \mathrm{H}-6), 6.91(1 \mathrm{H}, t, J=$ 7.6Hz, H-5), $6.39(1 \mathrm{H}, d, J=8.0 \mathrm{~Hz}, \mathrm{H}-4), 3.74-$ $3.67\left(1 \mathrm{H}, m, \mathrm{H}_{\mathrm{a}}-1^{\prime \prime}\right), 3.20-3.13\left(1 \mathrm{H}, m, \mathrm{H}_{\mathrm{b}}-1 "\right)$, $2.28\left(3 \mathrm{H}, \mathrm{s}, \mathrm{CH}_{3}-2\right), 2.25\left(3 \mathrm{H}, \mathrm{s}, \mathrm{CH}_{3}-3\right)$, 1.521.41 (2H, m, H-2"), 1.30-1.26 (2H, m, H-3"), 0.92 $\left(3 \mathrm{H}, t, J=7.2 \mathrm{~Hz}, \mathrm{CH}_{3}-4 "\right)$; El-MS $(\mathrm{m} / \mathrm{z}): 317\left(\mathrm{M}^{+}\right.$, 22.5\%), 141 (BP, 100\%), 120, 105, 77, 57, 51.

\section{$\mathrm{N}$-(2,3-Dimethylphenyl)-N-(1-methylpropyl) benzenesulfonamide(5e)}

Yield: $41 \%$; pinkish white solid; m.p. $92.6{ }^{\circ} \mathrm{C}$; Molecular formula: $\mathrm{C}_{18} \mathrm{H}_{23} \mathrm{NO}_{2} \mathrm{~S}$; Molecular weight: 317; Analysis. Calcd: C, 68.13; H, 7.25; $\mathrm{N}, 4.41 ; \mathrm{S}, 10.09$. Found: $\mathrm{C}, 66.65 ; \mathrm{H}, 6.88 ; \mathrm{N}$, 4.64; S, 10.34; IR $\left(\mathrm{cm}^{-1}\right) \quad U_{\max }: 3062(\mathrm{Ar} \mathrm{C}-\mathrm{H})$, 2973 (Alkane C-H), 1585 ( $\operatorname{Ar} \mathrm{C}=\mathrm{C}), 1337$ (S=O); ${ }^{1} \mathrm{H}-\mathrm{NMR}\left(400 \mathrm{MHz}, \mathrm{CDCl}_{3}, \delta\right.$ in ppm): $7.71(2 \mathrm{H}$, $\left.d, J=7.6 \mathrm{~Hz}, \mathrm{H}-2^{\prime}, \mathrm{H}-6{ }^{\prime}\right), 7.53(1 \mathrm{H}, t, J=7.2 \mathrm{~Hz}$, $\left.\mathrm{H}^{\prime} 4^{\prime}\right), 7.44\left(2 \mathrm{H}, t, J=7.6 \mathrm{~Hz}, \mathrm{H}-3^{\prime}, \mathrm{H}-5^{\prime}\right), 7.12$ $(1 \mathrm{H}, d, J=7.2 \mathrm{~Hz}, \mathrm{H}-6), 6.95(1 \mathrm{H}, t, J=7.6 \mathrm{~Hz}, \mathrm{H}-$ 5), $6.54(1 \mathrm{H}, d, J=8.0 \mathrm{~Hz}, \mathrm{H}-4), 4.29-4.23(1 \mathrm{H}$, $m, \mathrm{H}-1 "), 2.26\left(3 \mathrm{H}, s, \mathrm{CH}_{3}-2\right), 2.23\left(3 \mathrm{H}, s, \mathrm{CH}_{3}-\right.$ 3), $1.01(3 \mathrm{H}, d, J=6.8 \mathrm{~Hz}, \mathrm{H}-4 "), 0.90-0.85(2 \mathrm{H}$, $m, \mathrm{H}-2 "), 0.74\left(3 \mathrm{H}, t, J=7.2 \mathrm{~Hz}, \mathrm{CH}_{3}-3 "\right)$; El-MS $(\mathrm{m} / \mathrm{z}): 317\left(\mathrm{M}^{+}, 16.7 \%\right), 147$ (BP, 100\%), 141, 120, 105, 77, 57, 51.

\section{$\mathrm{N}$-(2,3-Dimethylphenyl)-N-pentylbenzenesul- fonamide (5f)}

Yield: $93 \%$; pinkish white solid; mp. $72.3{ }^{\circ} \mathrm{C}$; Molecular formula: $\mathrm{C}_{19} \mathrm{H}_{25} \mathrm{NO}_{2} \mathrm{~S}$; Molecular weight: 331; Analysis. Calcd: C, 68.88; H, 7.55; $\mathrm{N}, 4.22 ; \mathrm{S}, 9.66$. Found: $\mathrm{C}, 68.26 ; \mathrm{H}, 7.16 ; \mathrm{N}$, 4.52; S, 9.95;IR $\left(\mathrm{cm}^{-1}\right) u_{\text {max }}: 3069(\operatorname{Ar~C}-\mathrm{H}), 2959$ (Alkane C-H), 1585( $\mathrm{Ar} \mathrm{C}=\mathrm{C}), 1332(\mathrm{~S}=\mathrm{O}) ;{ }^{1} \mathrm{H}-$ NMR $\left(400 \mathrm{MHz}, \mathrm{CDCl}_{3}, \delta\right.$ in ppm): $7.65(2 \mathrm{H}, d, J$ $\left.=7.2 \mathrm{~Hz}, \mathrm{H}-2{ }^{\prime}, \mathrm{H}-6^{\prime}\right), 7.55\left(1 \mathrm{H}, t, J=7.6 \mathrm{~Hz}, \mathrm{H}-4^{\prime}\right)$, $7.45\left(2 \mathrm{H}, t, J=7.2 \mathrm{~Hz}, \mathrm{H}-3^{\prime}, \mathrm{H}-5^{\prime}\right), 7.08(1 \mathrm{H}, d, J$ $=7.2 \mathrm{~Hz}, \mathrm{H}-6), 6.91(1 \mathrm{H}, t, J=7.6 \mathrm{~Hz}, \mathrm{H}-5), 6.39$ $(1 \mathrm{H}, d, J=8.0 \mathrm{~Hz}, \mathrm{H}-4), 3.73-3.65\left(1 \mathrm{H}, m, \mathrm{H}_{\mathrm{a}}-1 "\right)$, 3.20-3.13 $\left(1 \mathrm{H}, m, \mathrm{H}_{\mathrm{b}}-1 "\right), 2.28\left(3 \mathrm{H}, s, \mathrm{CH}_{3}-2\right)$, $2.25\left(3 \mathrm{H}, s, \mathrm{CH}_{3}-3\right), 1.51-1.45(2 \mathrm{H}, m, \mathrm{H}-2 ")$, 1.26-1.20 (4H, m, H-3", H-4" ), $0.81(3 \mathrm{H}, t, J=$ $\left.6.8 \mathrm{~Hz}, \mathrm{CH}_{3}-5 "\right)$; El-MS $(\mathrm{m} / \mathrm{z}): 331\left(\mathrm{M}^{+}, 34.1 \%\right)$, 141, 120, 105, 77 (BP, 100\%), 71, 51.

\section{$\mathrm{N}$-(2,3-Dimethylphenyl)-N-(1-methylbutyl) benzenesulfonamide $(5 \mathrm{~g})$}

Yield: $89 \%$; light brownish liquid (extracted with chloroform); Molecular formula: $\mathrm{C}_{19} \mathrm{H}_{25} \mathrm{NO}_{2} \mathrm{~S}$; Molecular weight: 331; Analysis. Calcd: C, 68.88; $\mathrm{H}, 7.55$; N, 4.22; S, 9.66. Found: C, 68.23; H, 7.46; N, 4.19; S, 8.92;IR $\left(\mathrm{cm}^{-1}\right) u_{\max }: 3070$ (Ar CH), 2936 (Alkane C-H), 1580 ( $\mathrm{Ar} \mathrm{C}=\mathrm{C}), 1341$ 
$(\mathrm{S}=\mathrm{O}) ;{ }^{1} \mathrm{H}-\mathrm{NMR}\left(400 \mathrm{MHz}, \mathrm{CDCl}_{3}, \delta\right.$ in ppm): $7.71\left(2 \mathrm{H}, d, J=7.6 \mathrm{~Hz}, \mathrm{H}-2{ }^{\prime}, \mathrm{H}-6{ }^{\prime}\right), 7.53(1 \mathrm{H}, t, J$ $\left.=7.2 \mathrm{~Hz}, \mathrm{H}-4^{\prime}\right), 7.44\left(2 \mathrm{H}, t, J=7.6 \mathrm{~Hz}, \mathrm{H}-3^{\prime}, \mathrm{H}-5^{\prime}\right)$, $7.12(1 \mathrm{H}, d, J=7.2 \mathrm{~Hz}, \mathrm{H}-6), 6.95(1 \mathrm{H}, t, J=$ $7.6 \mathrm{~Hz}, \mathrm{H}-5), 6.64(1 \mathrm{H}, d, J=7.6 \mathrm{~Hz}, \mathrm{H}-4), 4.36-$ $4.33(1 \mathrm{H}, m, \mathrm{H}-1 "), 1.32-1.23(2 \mathrm{H}, m, \mathrm{H}-2 ")$, 1.15-1.12 (2H, m, H-3"), $1.01(3 \mathrm{H}, d, J=6.8 \mathrm{~Hz}$, $\left.\mathrm{CH}_{3}-5^{\prime \prime}\right), 0.77\left(3 \mathrm{H}, t, J=7.2 \mathrm{~Hz}, \mathrm{CH}_{3}-4 "\right)$; El-MS $(\mathrm{m} / \mathrm{z}): 331\left(\mathrm{M}^{+}, 1.1 \%\right), 141,120,105,77$ (BP, $100 \%), 71,56,51$.

\section{N-(2,3-Dimethylphenyl)-N-benzylbenzene- sulfonamide $(5 \mathrm{~h})$}

Yield: $88 \%$; shiny pinkish white solid; mp. 80.8 ${ }^{\circ} \mathrm{C}$; Molecular formula: $\mathrm{C}_{21} \mathrm{H}_{21} \mathrm{NO}_{2} \mathrm{~S}$; Molecular weight: 351; Analysis. Calcd: C, 71.79; H, 5.98; N, 3.98; S, 9.11. Found: C, 70.32; H, 5.72; N, 3.66; S, 9.26; IR (cm $\left.{ }^{-1}\right) U_{\text {max }}: 3054$ (Ar C-H), 2927 (Alkane C-H), 1585 ( $\mathrm{Ar} \mathrm{C}=\mathrm{C}), 1330(\mathrm{~S}=\mathrm{O}) ;{ }^{1} \mathrm{H}$ NMR (400 MHz, $\mathrm{CDCl}_{3}, \delta$ in ppm): $7.71(2 \mathrm{H}, d, J$ $\left.=7.6 \mathrm{~Hz}, \mathrm{H}-2{ }^{\prime}, \mathrm{H}-6^{\prime}\right), 7.59\left(1 \mathrm{H}, t, J=7.6 \mathrm{~Hz}, \mathrm{H}-4{ }^{\prime}\right)$, $7.48\left(2 \mathrm{H}, t, J=7.2 \mathrm{~Hz}, \mathrm{H}-3^{\prime}, \mathrm{H}^{-5}\right), 7.18-7.09(5 \mathrm{H}$, $m, \mathrm{H}-2 "$ to $\mathrm{H}-6 "), 7.01(1 \mathrm{H}, d, J=7.6 \mathrm{~Hz}, \mathrm{H}-6)$, $6.88(1 \mathrm{H}, t, J=7.6, \mathrm{H}-5), 6.41(1 \mathrm{H}, d, J=8.0 \mathrm{~Hz}$, $\mathrm{H}-4), 4.96\left(1 \mathrm{H}, d, J=13.6 \mathrm{~Hz}, \mathrm{H}_{\mathrm{a}}-7^{\prime \prime}\right), 4.24(1 \mathrm{H}$, $\left.d, J=13.6 \mathrm{~Hz}, \mathrm{H}_{\mathrm{b}}-7^{\prime \prime}\right), 2.14\left(3 \mathrm{H}, \mathrm{s}, \mathrm{CH}_{3}-2\right), 1.88$ $\left(3 \mathrm{H}, \mathrm{s}, \mathrm{CH}_{3}-3\right)$; El-MS $(\mathrm{m} / \mathrm{z}): 351\left(\mathrm{M}^{+}, 13.4 \%\right)$, 141, 120, 105, 91 (BP, 100\%), 77, 51.

\section{N-(2,3-Dimethylphenyl)-N-(2-chlorobenzyl) benzenesulfonamide $(5 \mathrm{i})$}

Yield: $98 \%$; pinkish white solid; mp. $109{ }^{\circ} \mathrm{C}$; Molecular formula: $\mathrm{C}_{21} \mathrm{H}_{20} \mathrm{CINO}_{2} \mathrm{~S}$; Molecular weight: 385; Analysis. Calcd: C, 65.45; H, 5.19; $\mathrm{N}, 3.63 ; \mathrm{S}$, 8.31. Found: C, 65.01; H, 5.38; N, 3.80; S, 7.71; IR (cm $\left.{ }^{-1}\right) U_{\max }: 3063(\mathrm{Ar} \mathrm{C}-\mathrm{H}), 2927$ (Alkane C-H), 1582 ( $\mathrm{Ar} \mathrm{C=C),} 1342(\mathrm{~S}=\mathrm{O}), 576$ (C-Cl); ${ }^{1} \mathrm{H}-\mathrm{NMR}\left(400 \mathrm{MHz}, \mathrm{CDCl}_{3}, \delta\right.$ in ppm): $7.72\left(2 \mathrm{H}, d, J=7.6 \mathrm{~Hz}, \mathrm{H}-2{ }^{\prime}, \mathrm{H}-6{ }^{\prime}\right), 7.60(1 \mathrm{H}, t, J$ $\left.=7.6 \mathrm{~Hz}, \mathrm{H}-4{ }^{\prime}\right), 7.49\left(2 \mathrm{H}, t, J=7.6 \mathrm{~Hz}, \mathrm{H}-3{ }^{\prime}, \mathrm{H}-5^{\prime}\right)$, $7.33\left(1 \mathrm{H}, d d, J=7.2,3.2 \mathrm{~Hz}, \mathrm{H}-3^{\prime \prime}\right), 7.19(1 \mathrm{H}, d d$, $J=6.4,2.8 \mathrm{~Hz}, \mathrm{H}-6 "), 7.13-7.09$ (2H, m, H-4", H5"), $7.00(1 \mathrm{H}, d, J=7.6 \mathrm{~Hz}, \mathrm{H}-6), 6.87(1 \mathrm{H}, t, J=$ $7.6 \mathrm{~Hz}, \mathrm{H}-5), 6.52(1 \mathrm{H}, d, J=7.6 \mathrm{~Hz}, \mathrm{H}-4), 5.08$ $\left(1 \mathrm{H}, d, J=14.0 \mathrm{~Hz}, \mathrm{H}_{\mathrm{a}}-7^{\prime \prime}\right), 4.53(1 \mathrm{H}, d, J=14.0$ $\left.\mathrm{Hz}, \mathrm{H}_{\mathrm{b}}-7^{\prime \prime}\right), 2.13\left(3 \mathrm{H}, \mathrm{s}, \mathrm{CH}_{3}-2\right), 1.91\left(3 \mathrm{H}, s, \mathrm{CH}_{3}-\right.$ 3); El-MS $(\mathrm{m} / \mathrm{z}): 387\left([\mathrm{M}+2]^{+}, 6.1 \%\right), 385\left(\mathrm{M}^{+}\right.$, 17.9\%), 141, 120, 125 (BP, 100\%), 105, 90, 77, 51.

\section{$\mathrm{N}-(2,3-$ Dimethylphenyl)-N-(4-chlorobenzyl) benzenesulfonamide (5j)}

Yield: $50 \%$; pinkish white solid; mp. $104.8^{\circ} \mathrm{C}$; Molecular formula: $\mathrm{C}_{21} \mathrm{H}_{20} \mathrm{CINO}_{2} \mathrm{~S}$; Molecular weight: 385; Analysis. Calcd for: C, 65.45; $\mathrm{H}$,
5.19; N, 3.63; S, 8.31. Found: C, 64.86; H, 5.45; $\mathrm{N}, 3.35 ; \mathrm{S}, 8.18$; IR $\left(\mathrm{cm}^{-1}\right) U_{\max }: 3066(\operatorname{Ar~C-H})$, 2923 (Alkane C-H), 1584 ( $\operatorname{Ar} \mathrm{C}=\mathrm{C}), 1342$ ( $\mathrm{S}=\mathrm{O})$, 581 (C-Cl); ${ }^{1} \mathrm{H}-\mathrm{NMR}$ (400 MHz, $\mathrm{CDCl}_{3}, \delta$ in ppm): $7.69\left(2 \mathrm{H}, d, J=7.6 \mathrm{~Hz}, \mathrm{H}-2^{\prime}, \mathrm{H}-6{ }^{\prime}\right), 7.59(1 \mathrm{H}, t, J$ $\left.=7.2 \mathrm{~Hz}, \mathrm{H}-4^{\prime}\right), 7.48\left(2 \mathrm{H}, t, J=7.6 \mathrm{~Hz}, \mathrm{H}-3^{\prime}, \mathrm{H}^{\prime} 5^{\prime}\right)$, $7.15(2 \mathrm{H}, d, J=8.4 \mathrm{~Hz}, \mathrm{H}-3 ", \mathrm{H}-5 "), 7.05(2 \mathrm{H}, d$, $J=8.4 \mathrm{~Hz}, \mathrm{H}-2 ", \mathrm{H}-6 "), 7.02(1 \mathrm{H}, d, J=8.0 \mathrm{~Hz}$, $\mathrm{H}-6), 6.88(1 \mathrm{H}, t, J=7.6 \mathrm{~Hz}, \mathrm{H}-5), 6.39(1 \mathrm{H}, d, J$ $=7.6 \mathrm{~Hz}, \mathrm{H}-4), 4.91\left(1 \mathrm{H}, d, J=13.6 \mathrm{~Hz}, \mathrm{H}_{\mathrm{a}}-7^{\prime \prime}\right)$, $4.21\left(1 \mathrm{H}, d, J=13.6 \mathrm{~Hz}, \mathrm{H}_{\mathrm{b}}-7^{\prime \prime}\right), 2.15(3 \mathrm{H}, s$, $\left.\mathrm{CH}_{3}-2\right)$, $1.89\left(3 \mathrm{H}, s, \mathrm{CH}_{3}-3\right)$; El-MS $(\mathrm{m} / \mathrm{z}): 387$ $\left([\mathrm{M}+2]^{+}, 5.2 \%\right), 385\left(\mathrm{M}^{+}, 15.4 \%\right), 141,120,125$ (BP, 100\%), 105, 90, 77, 51.

\section{$\mathrm{N}$-(2,3-Dimethylphenyl)-N-(4-bromobenzyl) benzenesulfonamide (5k)}

Yield: $98 \%$; off-white solid; mp. $106.3{ }^{\circ} \mathrm{C}$; Molecular formula: $\mathrm{C}_{21} \mathrm{H}_{20} \mathrm{BrNO}_{2} \mathrm{~S}$; Molecular weight: 430; Analysis. Calcd: C, 58.60; H, 4.65; $\mathrm{N}, 3.25 ; \mathrm{S}, 7.44$. Found: C, 58.19; H, 4.72; N, 3.41; S, 7.12; IR (cm $\left.{ }^{-1}\right) U_{\text {max }}: 3065(\mathrm{Ar} \mathrm{C}-\mathrm{H}), 2924$ (Alkane C-H), 1586 ( $\operatorname{Ar} \mathrm{C}=\mathrm{C}), 1342(\mathrm{~S}=\mathrm{O}), 585$ (C-Br); ${ }^{1} \mathrm{H}-\mathrm{NMR}\left(400 \mathrm{MHz}, \mathrm{CDCl}_{3}, \delta\right.$ inppm): 7.69 $\left(2 \mathrm{H}, d, J=7.2 \mathrm{~Hz}, \mathrm{H}-2^{\prime}, \mathrm{H}-6^{\prime}\right), 7.59(1 \mathrm{H}, t, J=$ $\left.7.6 \mathrm{~Hz}, \mathrm{H}-4^{\prime}\right), 7.48\left(2 \mathrm{H}, t, J=7.6 \mathrm{~Hz}, \mathrm{H}-3^{\prime}, \mathrm{H}-5^{\prime}\right)$, $7.30(2 \mathrm{H}, d, J=8.4 \mathrm{~Hz}, \mathrm{H}-3 ", \mathrm{H}-5 "), 7.02(1 \mathrm{H}, d$, $J=8.0 \mathrm{~Hz}, \mathrm{H}-6), 6.99(2 \mathrm{H}, d, J=8.4 \mathrm{~Hz}, \mathrm{H}-2 ", \mathrm{H}-$ 6"), $6.88(1 \mathrm{H}, t, J=7.6 \mathrm{~Hz}, \mathrm{H}-5), 6.39(1 \mathrm{H}, d, J=$ $8.0 \mathrm{~Hz}, \mathrm{H}-4), 4.89\left(1 \mathrm{H}, d, J=13.6 \mathrm{~Hz}, \mathrm{H}_{\mathrm{a}}-7 "\right)$, $4.20\left(1 \mathrm{H}, d, J=14.0 \mathrm{~Hz}, \mathrm{H}_{\mathrm{b}}-7^{\prime \prime}\right), 2.16(3 \mathrm{H}, s$, $\left.\mathrm{CH}_{3}-2\right), 1.90\left(3 \mathrm{H}, \mathrm{s}, \mathrm{CH}_{3}-3\right)$; El-MS $(\mathrm{m} / \mathrm{z}): 432$ $\left([\mathrm{M}+2]^{+}, 7.8 \%\right), 430\left(\mathrm{M}^{+}, 8.2 \%\right), 141,120,169$, 105, 90, 77 (BP, 100\%), 51.

\section{N-(2,3-Dimethylphenyl)-N-(phenylethyl)ben- zenesulfonamide (5I)}

Yield: $89 \%$; pinkish white solid; mp. $103.5^{\circ} \mathrm{C}$; Molecular formula: $\mathrm{C}_{22} \mathrm{H}_{23} \mathrm{NO}_{2} \mathrm{~S}$; Molecular weight: 365; Analysis. Calcd for: C, 72.32; $\mathrm{H}$, 6.30; N, 3.83; S, 8.76. Found: C, 71.71; H, 6.12; $\mathrm{N}, 3.95 ; \mathrm{S}, 8.90$; IR $\left(\mathrm{cm}^{-1}\right) U_{\max }: 3064(\operatorname{Ar~C}-\mathrm{H})$, 2922 (Alkane C-H), 1582 ( $\mathrm{Ar} \mathrm{C}=\mathrm{C}), 1342$ (S=O); ${ }^{1} \mathrm{H}-\mathrm{NMR}\left(400 \mathrm{MHz} \mathrm{CDCl}_{3}, \delta\right.$ inppm): $7.64(2 \mathrm{H}, d$, $\left.J=8.0 \mathrm{~Hz}, \mathrm{H}-2{ }^{\prime}, \mathrm{H}-6^{\prime}\right), 7.54(1 \mathrm{H}, t, J=7.2 \mathrm{~Hz}, \mathrm{H}-$ $\left.4^{\prime}\right), 7.44\left(2 \mathrm{H}, t, J=8.0 \mathrm{~Hz}, \mathrm{H}-3^{\prime}, \mathrm{H}-5{ }^{\prime}\right), 7.22(2 \mathrm{H}$, $d, J=7.6 \mathrm{~Hz}, \mathrm{H}-3 ", \mathrm{H}-5 "), 7.17(1 \mathrm{H}, t, J=6.8 \mathrm{~Hz}$, $\mathrm{H}-4 "), 7.11(1 \mathrm{H}, d, J=7.2 \mathrm{~Hz}, \mathrm{H}-6), 7.06(2 \mathrm{H}, d$, $J=7.2 \mathrm{~Hz}, \mathrm{H}-2 ", \mathrm{H}-6 "), 6.95(1 \mathrm{H}, t, J=7.6 \mathrm{~Hz}, \mathrm{H}-$ 5), $6.47(1 \mathrm{H}, d, J=8.0 \mathrm{~Hz}, \mathrm{H}-4), 4.00-3.93(1 \mathrm{H}$, $\left.m, \mathrm{H}_{\mathrm{a}}-8 "\right), 3.46-3.36\left(1 \mathrm{H}, m, \mathrm{H}_{\mathrm{b}}-8 "\right), 2.88-2.82$ (1H, m, $\left.\mathrm{H}_{\mathrm{a}}-7^{\prime \prime}\right), 2.68-2.62\left(1 \mathrm{H}, m, \mathrm{H}_{\mathrm{b}}-7^{\prime \prime}\right), 2.29$ $\left(3 \mathrm{H}, s, \mathrm{CH}_{3}-2\right), 2.21\left(3 \mathrm{H}, \mathrm{s}, \mathrm{CH}_{3}-3\right)$; El-MS (m/z): $365\left(\mathrm{M}^{+}, 25.8 \%\right), 141,120,105,104,77$ (BP, $100 \%), 51$. 


\section{$\mathrm{N}$-(2,3-Dimethylphenyl)- $\mathrm{N}$-allylbenzenesulfo- namide $(5 \mathrm{~m})$}

Yield: $88 \%$; brownish white solid; mp. $60.5^{\circ} \mathrm{C}$; Molecular formula: $\mathrm{C}_{17} \mathrm{H}_{19} \mathrm{NO}_{2} \mathrm{~S}$; Molecular weight: 301; Analysis. Calcd: C, 67.77; H, 6.31; $\mathrm{N}, 4.65 ; \mathrm{S}, 10.63$. Found: C, 66.83; H, 6.08; N, 4.30; S, 10.72;IR ( $\left.\mathrm{cm}^{-1}\right) u_{\max }: 3063($ Ar C-H), 2946 (Alkane C-H), 1580 (Ar $\mathrm{C}=\mathrm{C}), 1705$ (Alkene $\mathrm{C}=\mathrm{C}), 1341(\mathrm{~S}=\mathrm{O}) ;{ }^{1} \mathrm{H}-\mathrm{NMR}\left(400 \mathrm{MHz}, \mathrm{CDCl}_{3}, \delta\right.$ in ppm): $7.68\left(2 \mathrm{H}, d, J=7.6 \mathrm{~Hz}, \mathrm{H}-2^{\prime}, \mathrm{H}-6^{\prime}\right), 7.56$ $(1 \mathrm{H}, t, J=7.2 \mathrm{~Hz}, \mathrm{H}-4), 7.47(2 \mathrm{H}, t, J=7.6 \mathrm{~Hz}, \mathrm{H}-$ 3', H-5'), $7.07(1 \mathrm{H}, d, J=7.6 \mathrm{~Hz}, \mathrm{H}-6), 6.90(1 \mathrm{H}$, $t, J=7.6 \mathrm{~Hz}, \mathrm{H}-5), 6.39(1 \mathrm{H}, d, J=7.6 \mathrm{~Hz}, \mathrm{H}-4)$, 5.76-5.69 (1H, m, H-2"), $4.97(1 \mathrm{H}, d d, J=6.4$, $\left.2.8 \mathrm{~Hz}, \mathrm{H}_{\mathrm{b}}-3^{\prime \prime}\right), 4.30\left(1 \mathrm{H}, d d, J=7.6,2.8 \mathrm{~Hz}, \mathrm{H}_{\mathrm{a}}-\right.$ 3"), $3.87(2 \mathrm{H}, d, J=6.4 \mathrm{~Hz}, \mathrm{H}-1 "), 2.26(3 \mathrm{H}, s$, $\left.\mathrm{CH}_{3}-2\right)$, $2.23\left(3 \mathrm{H}, s, \mathrm{CH}_{3}-3\right)$; El-MS $(\mathrm{m} / \mathrm{z}): 301$ $\left(\mathrm{M}^{+}, 20.9 \%\right), 141,120,105,77$ (BP, 100\%), 51.

\section{Antibacterial activity}

The synthesized compounds were evaluated against two Gram-positive and four Gramnegative bacterial strains using ampicillin and ciprofloxacin as standards. (Table 1). The compounds showed moderate to significant activity against bacterial strains except compounds, 5a, 5d and $5 \mathrm{e}$. The compound 3 was active against one strain of gram-positive bacteria only. The preliminary screening showed that compound, $\mathbf{5 c}, \mathbf{5 f}, \mathbf{5 g}, \mathbf{5 i}, \mathbf{5 I}$ and $\mathbf{5 m}$ were the most active ones against gram-positive and compounds, $\mathbf{5 b}, \mathbf{5} \mathbf{f}, \mathbf{5} \mathbf{j}$ and $\mathbf{5 k}$ were more active against gram-negative bacterial strains.

\section{Anti-enzymatic activity}

The screening against $\alpha$-glucosidase enzyme demonstrated that the most of compounds were active with good inhibitory potential except $\mathbf{5 a}$, $\mathbf{5 b}, \mathbf{5 c}$ and $\mathbf{3}$ (Table-2). The molecules $\mathbf{5 g}$ and $\mathbf{5 j}$ were found to be excellent inhibitors with their $I_{50}$ values of $59.53 \pm 0.008$ and $55.31 \pm 0.01$ $\mu$ moles/L respectively, relative to acarbose a reference standard with $\mathrm{IC}_{50}$ value $38.25 \pm$ $0.12 \mu$ moles/L.

\section{Hemolytic activity}

The compounds $\mathbf{5 a}, \mathbf{5 b}, \mathbf{5 d}, \mathbf{5 h}$ and $\mathbf{5 j}$ exhibited cytotoxic activity with percent lysis of $27.2 \pm 0.24$, $22.4 \pm 0.21,21.7 \pm 0.68,18.2 \pm 0.56$ and $18.9 \pm$ 0.23 respectively, which was much lower than the positive control triton X-100 $(0.1 \% \mathrm{v} / \mathrm{v})$ which has percent lysis value of 100 . The compound $\mathbf{5 e}$ was the least cytotoxic in this series with percent lysis of $5.2 \pm 0.41$.

\section{DISCUSSION}

Sulfonamide $\mathbf{3}$ was obtained as off-white powder. Although its structure has been established by the x-ray crystallography [21] but more structural details are presented here for the first time. Its molecular formula, $\mathrm{C}_{14} \mathrm{H}_{15} \mathrm{NO}_{2} \mathrm{~S}$, was determined through CHNS analysis with percentage of elements as C: $64.03 \%$; $\mathrm{H}: 6.40 \%$; N: $5.52 \%$ and S: $11.73 \%$.

Table 1: MIC of synthesized compounds

\begin{tabular}{|c|c|c|c|c|c|c|}
\hline \multirow{2}{*}{ Compound } & \multicolumn{6}{|c|}{ MIC } \\
\hline & B. subtilis (+) & S. aureus (+) & E. coli (-) & S. sonnei (-) & S. typhi (-) & P. aeruginosa (-) \\
\hline 3 & $16.11 \pm 0.19$ & - & - & - & - & - \\
\hline $5 a$ & - & - & - & - & - & - \\
\hline $5 b$ & $11.46 \pm 0.22$ & - & $12.11 \pm 0.27$ & $19.83 \pm 0.04$ & $16.56 \pm 0.31$ & $11.7 \pm 0.32$ \\
\hline $5 c$ & $14.28 \pm 0.24$ & $11.36 \pm 0.20$ & $16.23 \pm 0.14$ & - & $13.01 \pm 0.18$ & $11.06 \pm 0.70$ \\
\hline $5 d$ & - & - & - & - & - & - \\
\hline $5 e$ & - & - & - & - & - & - \\
\hline $5 f$ & $17.40 \pm 0.28$ & $12.51 \pm 0.31$ & $11.36 \pm 0.00$ & $15.04 \pm 0.33$ & $13.17 \pm 0.18$ & $13.34 \pm 0.12$ \\
\hline $5 g$ & $18.52 \pm 0.00$ & $19.33 \pm 0.32$ & $16.13 \pm 0.57$ & $12.62 \pm 0.19$ & - & - \\
\hline $5 \mathrm{~h}$ & - & $15.52 \pm 0.09$ & $19.00 \pm 0.18$ & - & $16.33 \pm 0.28$ & $14.40 \pm 1.23$ \\
\hline $5 i$ & $10.78 \pm 0.09$ & $13.47 \pm 0.52$ & $18.86 \pm 0.01$ & - & - & $12.20 \pm 0.31$ \\
\hline $5 j$ & $12.52 \pm 0.73$ & - & $17.36 \pm 0.18$ & $11.86 \pm 0.12$ & $12.33 \pm 0.15$ & $13.22 \pm 0.50$ \\
\hline $5 k$ & - & $10.78 \pm 0.46$ & $16.54 \pm 0.38$ & $16.43 \pm 0.21$ & $10.89 \pm 0.11$ & $13.98 \pm 0.90$ \\
\hline 51 & $17.43 \pm 0.24$ & $12.52 \pm 0.11$ & $15.36 \pm 0.28$ & $18.09 \pm 0.83$ & - & $14.52 \pm 0.24$ \\
\hline $5 \mathrm{~m}$ & $10.10 \pm 0.29$ & $17.43 \pm 0.13$ & - & - & - & $14.55 \pm 0.02$ \\
\hline Ampicillin & $13.92 \pm 0.29$ & $11 \pm 0.01$ & $11.32 \pm 0.13$ & $13.11 \pm 0.11$ & $12.78 \pm 0.21$ & $16.86 \pm 0.31$ \\
\hline Ciprofloxacin & $8.36 \pm 0.12$ & $9.42 \pm 0.11$ & $8.21 \pm 0.02$ & $15.34 \pm 0.22$ & $7.59 \pm 0.11$ & $11.03 \pm 0.10$ \\
\hline
\end{tabular}


The molecular formula was also supported by $\mathrm{El}-\mathrm{MS}$, showing a $[\mathrm{M}]^{+}$ion peak at $\mathrm{m} / \mathrm{z} 261$ and by the integration curves in its ${ }^{1} \mathrm{H}$-NMR spectrum indicating the number of protons for each peak. The signals in ${ }^{1} \mathrm{H}$-NMR spectrum, appearing at $\delta$ 7.69 (d, J = 7.6Hz, 2H, H-2', H-6'), 7.53 (t, J = 7.6 $\left.\mathrm{Hz}, 1 \mathrm{H}, \mathrm{H}-4^{\prime}\right)$ and $7.41\left(\mathrm{t}, \mathrm{J}=7.6 \mathrm{~Hz}, 2 \mathrm{H}, \mathrm{H}-3^{\prime}, \mathrm{H}-\right.$ $\left.5^{\prime}\right)$ were typical for the protons of the benzenesulfonyl group present in the molecule. Furthermore, the signals resonating at $\delta 7.13(\mathrm{~d}$, $\mathrm{J}=7.6 \mathrm{~Hz}, 1 \mathrm{H}, \mathrm{H}-6), 6.96$ (t, $\mathrm{J}=7.6 \mathrm{~Hz}, 1 \mathrm{H}, \mathrm{H}-5$ ), $6.46(\mathrm{~d}, \mathrm{~J}=6.39 \mathrm{~Hz}, 1 \mathrm{H}, \mathrm{H}-4), 2.18(\mathrm{~s}, 3 \mathrm{H}, \mathrm{CH} 3-$ 2) and $1.91(\mathrm{~s}, 3 \mathrm{H}, \mathrm{CH} 3-3)$ were assigned to the protons of substituted aniline ring. FT-IR supported the structure by absorption bands at $3208(\mathrm{~N}-\mathrm{H}), 3042$ (Ar C-H), 2924 (Alkane C-H), $1583(\mathrm{Ar} \mathrm{C}=\mathrm{C})$ and $1324(\mathrm{~S}=\mathrm{O}) \mathrm{cm}^{-1}$. On the basis of above accumulative manifests, the structure of $\mathbf{3}$ was confirmed as $\mathrm{N}$-(2,3dimethylphenyl)benzenesulfonamide. Similarly, the structures of other compounds, 5a-m, were characterized.

The antibacterial activity results declared the compound $\mathbf{5 f}$ bearing aliphatic straight chain with moderate length at nitrogen atom was the most active molecule against all the bacterial strains used in this study. The compounds with sulfamoyl moiety have been introduced as valuable biologically active compounds and in accordance with our previous findings [13-16], the overview of synthesized compounds hereby showed that $\mathrm{N}$-substitution of sulfamoyl group with aliphatic chains and halogenated aromatic compounds presented best activity because of best fit to active site of enzyme and better $\pi-\pi$ interactions. The two molecules, $\mathbf{5 g}$ and $\mathbf{5 j}$, showed good activity against a-glucosidase probably because of branched long alkyl chain in $5 \mathrm{~g}$ and $\mathrm{p}$-substituted halogenated aralkyl group in $\mathbf{5 j}$, presented better binding interactions with the active site of enzyme. The compound $5 e$ was the least cytotoxic in this series but it was inferred that all the compounds can be used as possible therapeutic agents.

\section{CONCLUSION}

All the compounds have been structurally identified by spectral data and screened for antibacterial, a-glucosidase and hemolytic analysis. The synthesized compounds exhibit cytotoxicity and antibacterial potentials.

\section{REFERENCES}

1. Naseer M, Chohan M, Zahid $H$. Synthesis, characterization and reactivity towards first-row $d$ transition metals and biological significance of new pyridinyl derived $\mathrm{N}$-substituted sulfonamides. Appl Organometal Chem 2007; 21: 826-835.

2. Rambabu N, Modi VB, Goswami KJ. Synthesis and characterization of N-(4-(4-chlorophenyl)-6-(3,4dimethylphenyl)pyrimidin-2-yl)-4-(2,6-diphenyl-4-thioxo2H-1,3,5-oxadiazin-3(4H)-yl)benzenesulfonamide. Der Pharma Chemica 2012; 4(1): 511-516.

3. Caballero RD, Torres-Lapasió JR, Baeza-Baeza JJ, García-Alvarez-Coque MC. Micellar chromatographic procedure with direct injection for the determination of sulfonamides in milk and honey samples. J Liq Chrom Rel Technol 2001; 24(1): 117-131.

4. Lackner H, Doring G, Garre K. NMR spectroscopic identification of sulfonamides from urine. Forensic Sci 1974; 4: 219-231.

5. Chen A, Wang $G$, Cao $Q$, Wang $Y$, Zhang $Z$, Sun $Y$, Wang $H$, Xu C, Zhou $Q$, Han P, Liu M, Yang $Y$, Xing $W$, Mitchelson K, Cheng J. Development of an antibody hapten-chip system for detecting the residues of multiple antibiotic drugs. J Forensic Sci 2009; 54(4): 953-960.

6. Rieder MJ, Krause R, Bird IA. Time-course of toxicity of reactive sulfonamide metabolites. Toxicol 1995; 95: 141 146.

7. Chiba S. Molecular mechanism of $\alpha$-glucosidase and glucoamylase. Biosci Biotech Biochem 1997; 61: 12331239.

8. Baron AD. Postprandial hyperglycemia and alphaglucosidase inhibitor. Diabetes Res Clin Pr 1998; 40(Suppl.):51-55.

9. Lebovitz HE. Alpha-glucosidase inhibitors. Endocrinology and Metabolism. Clin North Am 1997; 26: 539-551.

10. Chapdilaine P, Tremblay RR, Dube JY. p-Nitrophenol- $\alpha$ $D$-Glucopyranoside as substrate for measurement of maltase activity in human semen. Clin Chem 1978; 24 : 208-211.

11. Sharma $P$, Sharma JD. In vitro hemolysis of human erythrocytes by plant extracts with antiplasmodial activity. J Ethnopharmacol 2001; 74: 239-243.

12. Dausset J, Contu L. Drug induced hemolysis. Ann Rev Med 1967; 18: 55-70.

13. Abbasi MA, Aziz-ur-Rehman, Muhmood T, Khan KM, Ashraf M, Ejaz SA, Arshad S. Synthesis structural characterization and biological screening of various sulfa drugs derived from 2-anisidine. J Chem Soc Pak 2013; 35(2): 404-410.

14. Abbasi MA, Ahmad S, Aziz-ur-Rehman, Rasool S, Khan $K M$., Ashraf $M$, Nasar $R$, Ismail $T$. Sulfonamide derivatives of 2-amino-1-phenylethane as suitable cholinesterase inhibitors. Trop J Pharm Res 2014; 13(5): 739-745.

15. Abbasi MA, Saeed A, Aziz-ur-Rehman, Khan KM, Ashraf $M$, Ejaz SA. Synthesis of brominated 2-phenitidine derivatives as valuable inhibitors of cholinesterases for the treatment of Alzheimer's disease. Iran J Pharm Res 2014; 13(1): 87-94.

16. Irshad M, Abbasi MA., Aziz-ur-Rehman, Rasool $S$, Siddiqui SZ, Ahmad I, Ashraf M, Lodhi MA, and Jamal $S B$. $O$ - and $N$-Substituted derivatives of planetol as 
valuable bioactive compounds. Asian J Chem 2014; 26 (4): 1151-1160.

17. Kaspady M, Narayanaswamy VK, Raju M, Rao GK. Synthesis, antibacterial activity of 2,4-disubstituted oxazoles and thiazoles as bioesters. Lett Drug Des Discov 2009; 6: 21-28.

18. Yang CR, Zang Y, Jacob MR, Khan SI, Zhang YJ, Li XC. Antifungal activity of $C-27$ steroidal saponins. Antimicrob Agents Ch 2006; 50(5): 1710-1714.
19. Powell WA, Catranis CM, Maynard CA. Design of selfprocessing antimicrobial peptide for plant protection. Lett Appl Microbiol 2000; 31(2): 163-168.

20. World Medical Association. World Medical Association Declaration of Helsinki; Ethical principles for medical research involving human subjects. Clin Rev Edu 2013; 310(20): 2191-2194.

21. Gowda BT, Foro S, Babitha KS, Fuess H. N-(2,3Dimethylphenyl)benzenesulfonamide. Acta Cryst 2009; E65: 0366. 